\title{
Heat and Hydrothermal Treatment on the Microstructure Evolution of Plasma Sprayed Hydroxyapatite Coatings Reinforced with
}

\section{Graphene Nanoplatelets}

\author{
Jia Ren, Dong Zhao, Fei Qi, Yongguang Wang, Yao Chen* \\ School of Mechanical and Electric Engineering \& Collaborative Innovation Center of Suzhou \\ Nano Science and Technology, Soochow University, 215123 Suzhou, China \\ * Correspondence. chenyao@ suda.edu.cn; Tel.:+86 52167581872
}

\begin{abstract}
Recent advances and demands in clinical applications drive a large amount of research to hydroxyapatite (HA) composite coatings fabricated by plasma spray. However, lower degree of HA crystallinity related to high temperature exposure in plasma spray usually leads to rapid weakening and disintegration of HA coatings and often promotes inflammatory responses in the surrounding tissue. In this research, graphene nanosheet (GNS) reinforced HA coatings were fabricated using plasma spray and followed by heat and hydrothermal treatment (hereafter referred to as thermal treatment). The addition of GNSs resulted in competing phenomenon to influence HA crystallinity viz. increased portion of the partially melted/unmelted zones and higher cooling rate during splat formation, leading to slight increase in HA crystallinity $(\sim 46.0-51.3 \%)$ in the as-sprayed coating. XRD and FTIR results showed that thermal treatment was capable of inducing significant transformation of amorphous HA to the crystalline form and removing other foreign non-HA compounds through regaining $\mathrm{OH}^{-}$ion, and therefore $\mathrm{HA}$ coatings displayed 45.5-47.1\% improvements in HA crystallinity regardless of addition or not of the GNS nanofillers. Microstructure observations revealed that the residual partially melted/unmelted zone of the thermally treated GNS/HA coating was significantly decreased in size, though thermal treatment enabled microcrack propagation due to
\end{abstract}


stresses caused by crystallisation and phase transformations. More importantly, the added GNSs contributed greatly to the significant increase in surface nanoroughness of the thermally treated HA coatings owing to the fact that much more structural defects along with the GNSs mainly induced by thermal treatment might act as nucleation sites to accelerate HA nanoparticle precipitation, which would be beneficial for the improved adhesion strength of the osteoblast cells on the coating surface.

Keywords: graphene; hydroxyapatite; crystallinity; surface roughness; heat and hydrothermal treatment; plasma spray 


\section{Introduction}

Hydroxyapatite $\left(\mathrm{Ca}_{10}(\mathrm{PO} 4)_{6}(\mathrm{OH})_{2}, \quad \mathrm{HA}\right), \quad$ one such type of calcium phosphate-based bioceramics, has been considered for various biomedical applications in dentistry and orthopedics owing to its chemical resemblance to the mineral component of the natural bones, excellent biocompatibility and osteoconductivity [1-3]. In spite of the excellent properties as biomaterials, the intrinsic brittleness of HA limits its clinical applications such as load-bearing implants [4]. As well recognized recently, the most promising way to overcome these problems is to coat $\mathrm{HA}$ on a titanium alloy implant, which is expected to achieve the combination of high mechanical strength of the titanium alloys with the excellent biocompatibility and bioactivity of HA, and is therefore suitable for implants in load-bearing applications $[5,6]$.

To further improve mechanical properties of HA coating itself, HA composite coatings with a second phase (usually ceramic particles such as $\mathrm{Al}_{2} \mathrm{O}_{3}, \mathrm{ZrO}_{2}$, and $\mathrm{TiO}_{2}$ ) have been extensively explored [7-12]. Despite significant improvement achieved in fracture toughness of these HA composite coatings, it is necessary to note that the addition contents of these ceramic particles, basically biologically inert, are usually as high as $30-50 \%$ weight fraction, inevitably leading to degradation of the excellent bioactivity associated with monolithic HA. Hence, an ideal reinforcement in HA coatings should possess significant toughening effect even with smaller amount additions to avoid negative influence on the bioactivity/osteointegration of HA [13]. In recent years, carbon nanotubes (CNTs) and graphene have been considered as 
promising nanofillers in ceramics to improve their fracture toughness $[14,15]$ due to their excellent mechanical properties, which open up a new material-design avenue to HA composites/coatings. Previous studies reported that the fracture toughness showed a $~ 56 \%$ improvement with a CNT addition of 4.0 wt.\% (weight percentage) in a plasma sprayed HA composite coating [16,17]. However, the biocompatibility of CNTs remains controversial, though some studies ascribed the cytotoxicity of CNTs to the presence of metallic catalyst particles rather than CNT itself [18]. Unlike CNTs, graphene can be synthesized in such pure ways as chemical vapor deposition, micromechanical exfoliation of graphite [19]. Our previous preliminary studies have demonstrated that graphene nanosheet (GNS) reinforced HA composite displayed $~ 80 \%$ improvement in fracture toughness at 1.0wt.\% GNS concentration and exhibited enhanced in vitro biocompatibility [20,21].

Among various coating techniques, plasma spray is an industrially versatile surface coating technique owing to the cost efficiency and simplicity of the process [18], and it is the only technique which has been approved clinically by Food and Drug Administration (FDA), USA [18]. However, it should be noted that some common problems, i.e., the formation of foreign phases such as $\alpha$-and $\beta$ tricalcium phosphate ( $\alpha$-TCP, $\beta$-TCP), tetracalcium phosphate (TTCP), $\mathrm{CaO}$, amorphous calcium phosphate (ACP), are related with plasma spray due to high temperature involved in the process $[22,23]$, which inevitably leads to lower degree of HA crystallinity. These foreign phases have been reported to greatly influence the mechanical properties and the biological behavior of the coating system, because ACP behaves the highest 
dissolution rate and crystalline $\mathrm{HA}$ is much more stable than other calcium compounds such as $\alpha$-TCP, $\beta$-TCP and TTCP [24]. To increase the content of crystalline HA in the as-sprayed coatings, post heat treatment has been developed recently [25]. Previous research found that the content of crystalline HA of the as-sprayed coatings increased from heat treatment temperature of $\sim 500{ }^{\circ} \mathrm{C}$ and reached a maximum (about $70 \%$ ) at temperature of $\sim 700{ }^{\circ} \mathrm{C}$, then decreased gradually with further increasing temperature [26-28]. However, it should be noted that heat treatment only facilitates ACP back to crystalline HA, but the decomposition products of HA cannot be retransformed back to crystalline HA due to the lack of $\mathrm{H}_{2} \mathrm{O}$ [29].

Herein, the aim of the present work was to fabricate GNS reinforced hydroxyapatite composite coatings through plasma spray, and to explore the effects of the addtion of GNS nanofillers, heat and hydrothermal treatment on the microstructure evolution of plasma sprayed HA composite coatings.

\section{Material and methods}

HA nanorods (purity: 99\%; diameter: $20 \mathrm{~nm}$ ) from Emperor Nano Material Company (Nanjing, China) and GNSs (purity: 99\%; thickness: $\sim 5 \mathrm{~nm}$, diameter: $\sim 2$ $\mu \mathrm{m})$ from XianFeng Nano Material Company (Nanjing, China) were employed in this research. Detailed information on the mixing of GNS and HA powders can be found in elsewhere [21]. The compositions chosen here were pure HA, 1.0wt.\%GNS/HA and 2.0wt.\%GNS/HA. Spray drying was employed to obtain microsized agglomerates with a size of $40-75 \mu \mathrm{m}$ and good flowability for plasma spray, some GNSs were observed to locate on the surface of the agglomerated composite powders [30]. 
Prior to plasma spray, Ti-6Al-4V substrates were grit blasted using $\mathrm{Al}_{2} \mathrm{O}_{3}$ particles and followed by being cleaned with acetone. Plasma spray was performed using SG 100 gun (Praxair Surface Technology, Danbury, CT), in which 20-25 kW plasma power and 600-700 A plasma-gun current were used with a powder feeding rate of $\sim 4.5 \mathrm{~g} / \mathrm{min}$ and a standoff distance of $100 \mathrm{~mm}$. Argon was used as the primary gas (32 slpm) and helium as an auxiliary gas (28 slpm). Argon was also used as powder carrier gas $(8 \mathrm{slpm})$. To reduced ACP content and to accelerate the transformation of the decomposition products back to HA, the as-sprayed coatings were heat treated at $600{ }^{\circ} \mathrm{C}$ for $2 \mathrm{~h}$ followed by hydrothermal treatment at $120{ }^{\circ} \mathrm{C}$ for $2 \mathrm{~h}$ in milli-Q water (hereafter referred to as thermal treatment in this research).

The cross-section of the as-sprayed coatings with and without thermal treatment were metallographically polished, and microstructural characterizations were revealed using field emission scanning electron microscopy (FESEM, Hitachi S-4700, Japan). X-ray diffraction (XRD, X'Pert-ProMRD, Holland) with $\mathrm{Cu} \mathrm{K} \alpha$ radiation was conducted to analyze the phase constituents and HA crystallinity of the coatings using a scanning rate of $5 \% \mathrm{~min}$, in which HA crystallinity of the coatings was calculated using the following relation [31]: Percentage of crystallinity $=A p / A c 100 \%$, where $A_{P}$

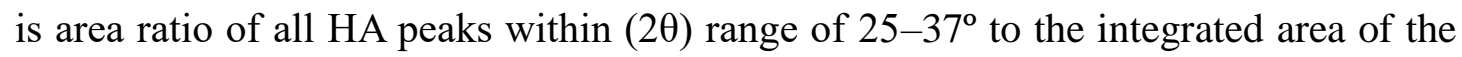
most intensive HA peak within (20) range of $31.4-31.9^{\circ}$ of HA powder and $A_{C}$ is the area ratio of all HA peaks within $(2 \theta)$ range of $25-37^{\circ}$ to the integrated area of the most intensive HA peak within (20) range of $31.4-31.9^{\circ}$ of respective HA coatings. Micro-Raman spectroscopy (Renishaw, UK) with an Argon ion laser of wavelength 
$633 \mathrm{~nm}$ and an acquisition time of $10 \mathrm{~s}$ was employed to confirm the existence of GNSs in the HA composite coatings. Fourier transform infrared (FTIR) spectroscope analysis (VERTEX 70, Bruker Optics, USA) was performed to characterize the $\mathrm{OH}-$ groups on the coatings, in which the infrared spectrum with the scan number of 32 was adopted with the scan range $4000-400 \mathrm{~cm}^{-1}$. In order to check the effects of thermal treatment and the addition of GNSs on the surface roughness, the top surface of the as-sprayed coatings was carefully polished and followed by thermal treatment. Then, Atomic force microscopy (AFM; Dimension Icon, Bruker, USA) was used to measure the surface roughness of each sample, in which scanning area of $10 \mu \mathrm{m} \times 10$ $\mu \mathrm{m}$ and scanning frequency of $2.5 \mathrm{~Hz}$ were employed.

\section{Results and discussion}

SEM images of the polished cross-section of the as-sprayed coatings are shown in Figure 1. Irrespective of addition or not of the GNS nanofillers, the coating-substrate interfaces were clear and no microcracks could be observed near the interface, implying that the adhesive strength of the coating to the substrates is high. In contrast to compact and dense microstructure of the as-sprayed HA coating was (Figure 1a), it is clear that some craters were observed in the GNS/HA coatings, as pointed out by the white arrows in Figure $1 \mathrm{~b}$ and 1c, and the number of craters increased with the added GNSs. In comparison, as shown in Figure 2, it was found that the thermal treatment resulted in substantial reduction in the crater number of the GNS/HA coatings. 

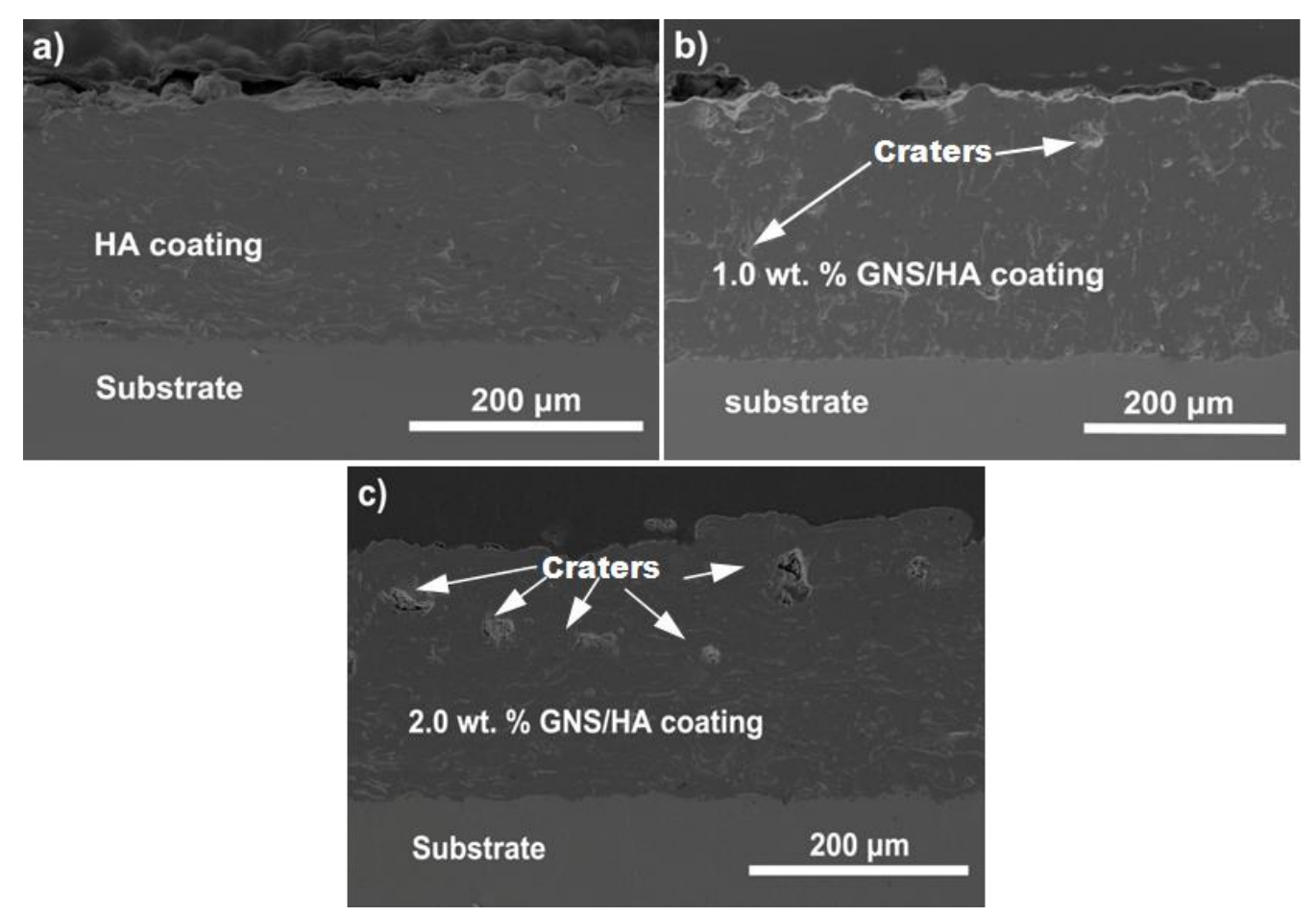

Figure 1. SEM images of cross-sectional view for plasma sprayed coatings of (a) HA coating, (b) $1.0 \mathrm{wt} \%$ GNS/HA coating and (c) $2.0 \mathrm{wt} \%$ GNS/HA coating.
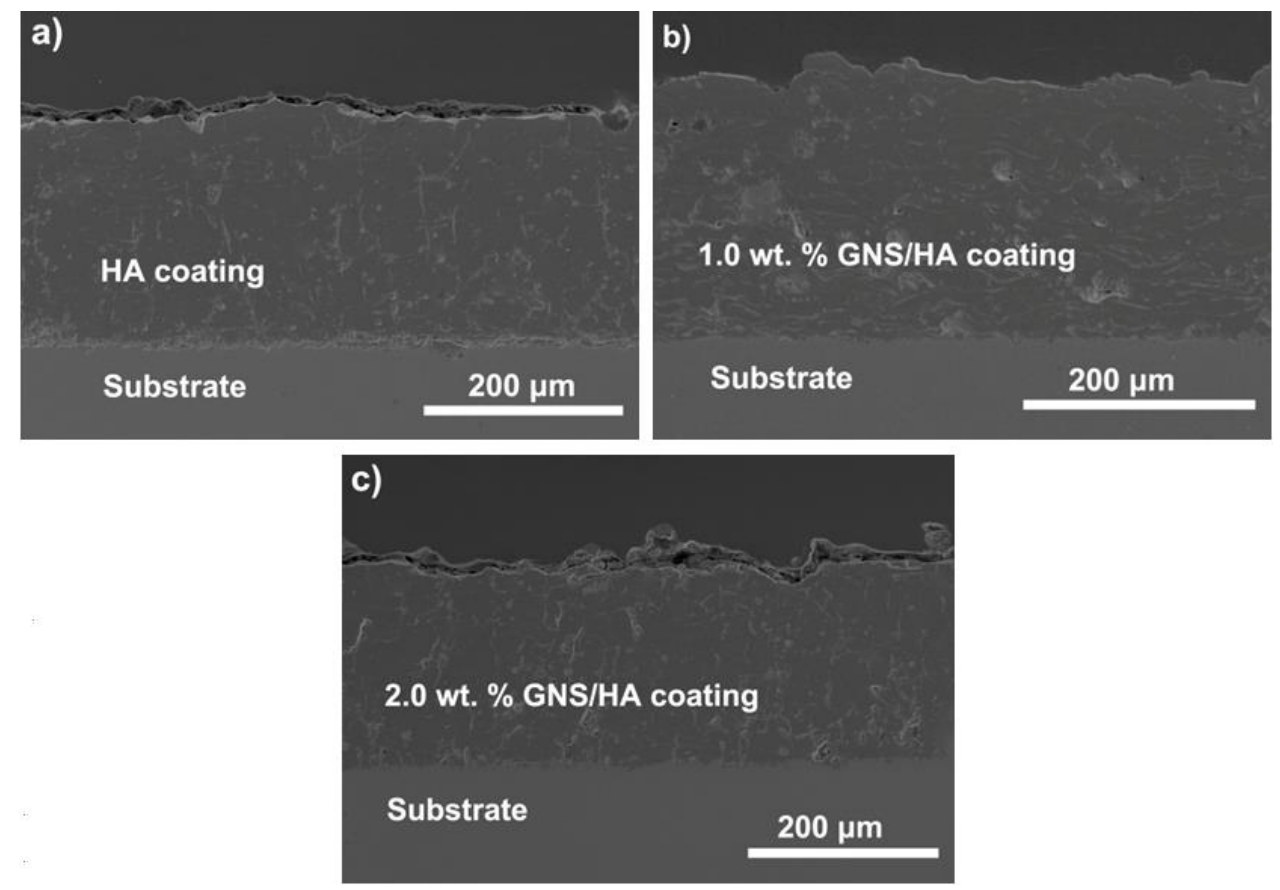

Figure 2. SEM images of cross-sectional view for thermally treated of (a) HA coating, (b) $1.0 \mathrm{wt} \% \mathrm{GNS} / \mathrm{HA}$ coating and (c) $2.0 \mathrm{wt} \% \mathrm{GNS} / \mathrm{HA}$ coating. 

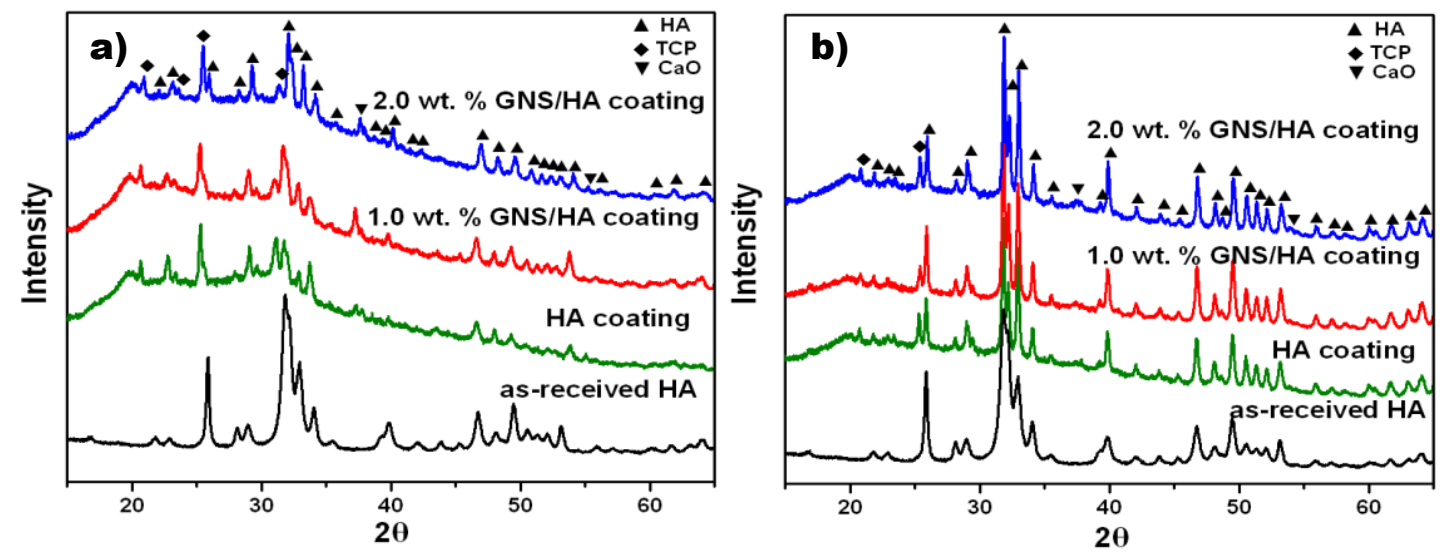

Figure 3. XRD results of (a) plasma sprayed HA and GNS/HA coatings and (b) thermally treated HA and GNS/HA coatings.

Figure 3a shows XRD results of the as-sprayed HA and GNS/HA coatings, it is clear that the main phases of the as-sprayed coatings were $\mathrm{HA}$, TCP and $\mathrm{CaO}$, and a "hump" centered in $2 \theta=20-35^{\circ}$ was observed due to the presence of ACP. The major reason for the formation of amorphous phases is the extremely high cooling rates (nearly $10^{7}-10^{8} \mathrm{~K} / \mathrm{s}$ ) associated with the molten droplets being impacted on the substrate during deposition. In general, HA usually decomposes into a mixture of TCP and TTCP in the temperature range of $\sim 1000-1450^{\circ} \mathrm{C}$ [24]. Nevertheless, the absence of TTCP and the presence of $\mathrm{CaO}$ in all as-sprayed coatings in this research implies that TTCP decomposition occurred, i.e., $\mathrm{Ca}_{4}\left(\mathrm{PO}_{4}\right)_{2} \mathrm{O} \stackrel{1550-1630^{\circ} \mathrm{C}}{\rightarrow} 4 \mathrm{CaO}+\mathrm{P}_{2} \mathrm{O}_{5}$, which is likely due to high-temperature exposure of feedstock materials during plasma spray. Meanwhile, $\mathrm{P}_{2} \mathrm{O}_{5}$ should be eliminated from the coatings as gas because of its low boiling point $\left(\sim 380^{\circ} \mathrm{C}\right)$. It is of importance to note that HA crystallinity of the as-sprayed HA coating was as low as $\sim 46.0 \%$, while the incorporation of GNSs slightly improved the content of crystalline HA in the composite coatings ( $49.2 \%$ for $0.5 \mathrm{wt} . \% \mathrm{GNS} / \mathrm{HA}$ coating and $\sim 51.3 \%$ for $1.0 \mathrm{wt} \% \mathrm{GNS} / \mathrm{HA}$ coating). As shown in 
Figure $3 b$, it is evident that the amorphous hump was significantly decreased after thermal treatment, more sharp and high intensity peaks were observed. Nevertheless, the existence of $\mathrm{TCP}$ and $\mathrm{CaO}$ in the thermally treated coating was also found, implying that the by-products of HA decomposition incompletely retransformed back to crystalline HA. Fortunately, thermal treatment significantly induced higher HA crystallinity in the as-sprayed coating, i.e., the calculated crystallinities were $\sim 67.7 \%$, $\sim 71.6 \%$ and $\sim 75.4 \%$ for HA coating, $0.5 \mathrm{wt} . \% \mathrm{GNS} / \mathrm{HA}$ coating and 1.0wt.\%GNS/HA coating, respectively. Above results strongly indicate that the addition of GNSs contributes to slightly increase in HA crystallinity, and thermal treatment is capable of significant improving the content of crystalline HA of the as-sprayed HA coatings. As well known, the most common level of HA crystallinity in the as-sprayed HA coatings for biomedical use is about $65-70 \%$, the presence of a small amount of amorphous HA on the coating surface may promote beneficial physiological activity [24].

Though ultrosonication of as-received GNS agglomerates in distilled water with sodium dodecyl-benzene sulphonate (SDBS) was employed to achieve GNS homogeneous distribution in this research, it is rather difficult to completely avoid GNS agglomeration due to their lager surface area and van de Walls force between them, especially in the case of higher addition content of GNSs in the powder mixture. Owing to very high thermal conductivity of GNSs $(\sim 5300 \mathrm{~W} / \mathrm{mK})[32]$, it is reasonable to envisage that the addition of GNSs might affect the degree of melting of the in-flight feedstock composite powders, i.e., GNS-rich regions in the feedstock composite powders would soak excess amount of heat from plasma plume than that of 
GNS-depleted regions. Hence, GNS agglomeration must result in much more GNS-depleted regions, it in turn leads to an increase in the portion of the partially melted or unmelted zones in the as sprayed coatings. These partially melted/unmelted zones present in the GNS/HA coatings followed by the grinding and polishing in the sample preparation ascribes to the observed craters on the cross-sections of the as-sprayed HA composite coatings (Figure 1). An increase in the partially melted/ unmelted zones might be beneficial to limit HA decomposition and ACP formation to some extent. On the other hand, in the case of a GNS/HA droplet impacting upon the substrate or previously deposited layer, the added GNSs with superior thermal conductivity and larger contact area are expected to impart a droplet higher cooling rate when it comes into contact with the substrate or previously deposited layer, and subsequently increasing the propensity for ACP formation. Clearly, the addition of GNSs resulted in competing phenomenon to influence HA crystallinity viz. increased portion of the partially melted/unmelted zones and higher cooling rate during splat formation, corresponding to the observations that the content of HA crystallinity in the as-sprayed coating increases with the addition content of GNSs.
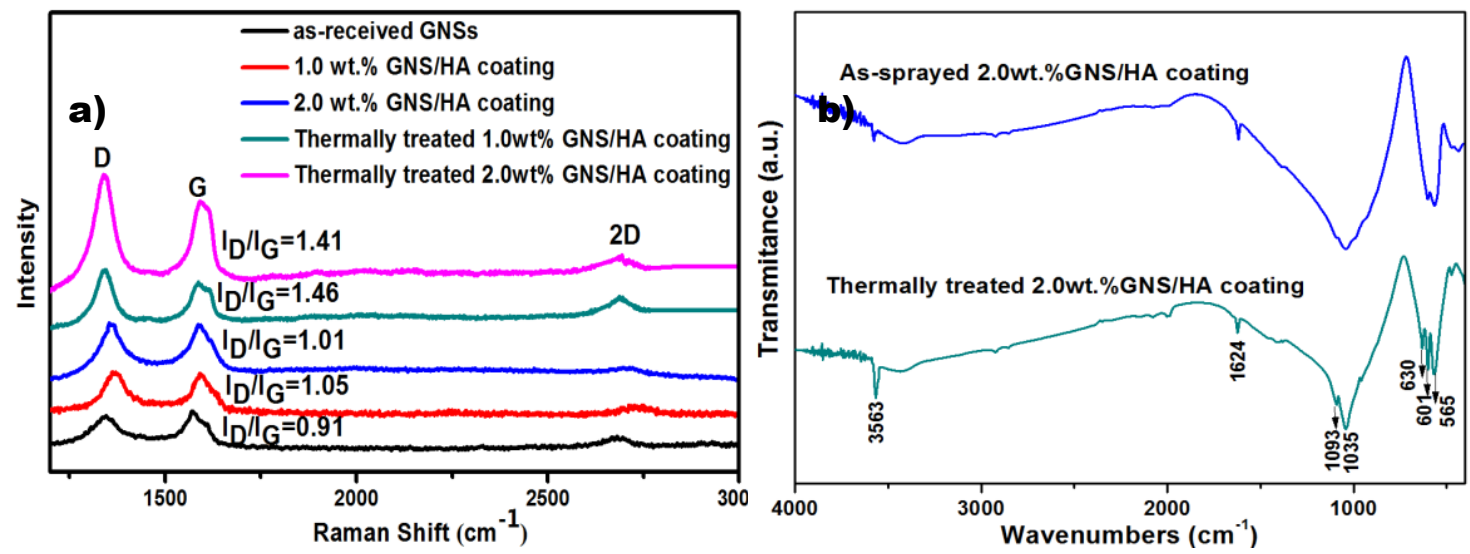

Figure 4. (a) Raman spectra of GNS/HA coatings with and without thermal treatment 
and (b) FTIR spectra of a $2.0 \mathrm{wt} \% \mathrm{GNS} / \mathrm{HA}$ coating with and without thermal treatment.

Raman measurement was performed to confirm the existence of GNSs. Figure 4a depicts the presence of $D, G$ and $2 D$ peaks, in which $G$ peak is from the in-plane C-C bond stretching in graphene, D peak is related to the defects in the structure, and 2D peak is associated with few-layered graphene structure $[33,34]$. The presence of $\mathrm{G}$ and $2 \mathrm{D}$ peaks in the composite coatings before and after thermal treatment confirmed that the short exposure (milliseconds) to high temperature during plasma spray and long exposure $(2 \mathrm{~h})$ to medium temperature $\left(600{ }^{\circ} \mathrm{C}\right)$ in the process of thermal treatment were not sufficient for the oxidation and destruction of GNSs, the inert carrier gas (argon) during plasma spray was also expected to create a shroud over the in-flight particles to protect the GNSs from oxidation. It is well known that intensity ratio of $D$ peak and $G$ peak $\left(\mathrm{I}_{\mathrm{D}} / \mathrm{I}_{\mathrm{G}}\right)$ is often used to quantify the degree of structural defects in graphene. As shown in Figure $4 a$, the $I_{D} / I_{G}$ values of as-sprayed 1.0wt.\%GNS/HA coating (1.05) and as-sprayed 2.0wt.\%GNS/HA coating (1.01) were slightly higher than that of as-received GNSs (0.95), illustrating that plasma spray introduced structural defects into GNSs. Meanwhile, in the case of thermally treated GNS/HA coatings, it should be noted that the $\mathrm{I}_{\mathrm{D}} / \mathrm{I}_{\mathrm{G}}$ value significantly increased to 1.46 for 1.0 wt. $\%$ GNS/HA coating and 1.41 for 1.0 wt.\% GNS/HA coating. These results strongly demonstrate that long exposure $(2 \mathrm{~h})$ during thermal treatment $\left(600{ }^{\circ} \mathrm{C}\right)$ induced much more structural defects into these incorporated GNSs, which was likely due to the relatively low oxidation temperature of graphene $\left(\sim 600^{\circ} \mathrm{C}\right)$ [35]. The survival of GNSs and regaining of $\mathrm{OH}^{-}$ion after thermal treatment was analyzed 
through FTIR spectra, as shown in Figure 4b. The survived GNPs were also revealed by the bands at 1624 , which can be assigned to the $\mathrm{C}=\mathrm{C}$ stretching vibration [36]. The $\mathrm{OH}^{-}$bands at $630 \mathrm{~cm}^{-1}$ and $3563 \mathrm{~cm}^{-1}$, and $\mathrm{PO}^{3-}{ }_{4}$ peaks at 565, 601, 1038, and 1093 $\mathrm{cm}^{-1}$ were clearly observed to become more sharper and prominent in the case of a GNS/HA composite coating followed by thermal treatment, sufficiently illustrating that thermal treatment is effective in regaining $\mathrm{OH}^{-}$ion and removing other foreign non-HA compounds. As for the transformation of foreign phases within the as-sprayed HA coatings, Chen et al. [37] suggested that TCP could be developed to HA phase by a series of hydrolytic reactions during thermal treatment as follows:

$10 \mathrm{Ca}_{3}\left(P O_{4}\right)_{2}+6 \mathrm{H}_{2} \mathrm{O}(\mathrm{g}) \rightarrow 3 \mathrm{Ca}_{10}\left(\mathrm{PO}_{4}\right)_{6}(\mathrm{OH})_{2}+2 \mathrm{H}_{3} \mathrm{PO}_{4}$

They also suggested that $\mathrm{CaO}$ converts into HA mostly by the following process [37]:

$\mathrm{CaO}+3 \mathrm{H}_{2} \mathrm{O}(\mathrm{g}) \rightarrow \mathrm{Ca}(\mathrm{OH})_{2}$

$10 \mathrm{Ca}(\mathrm{OH})_{2}+6 \mathrm{H}_{3} \mathrm{PO}_{4} \rightarrow \mathrm{Ca}_{10}\left(\mathrm{PO}_{4}\right)_{6}(\mathrm{OH})_{2}+18 \mathrm{H}_{2} \mathrm{O}$

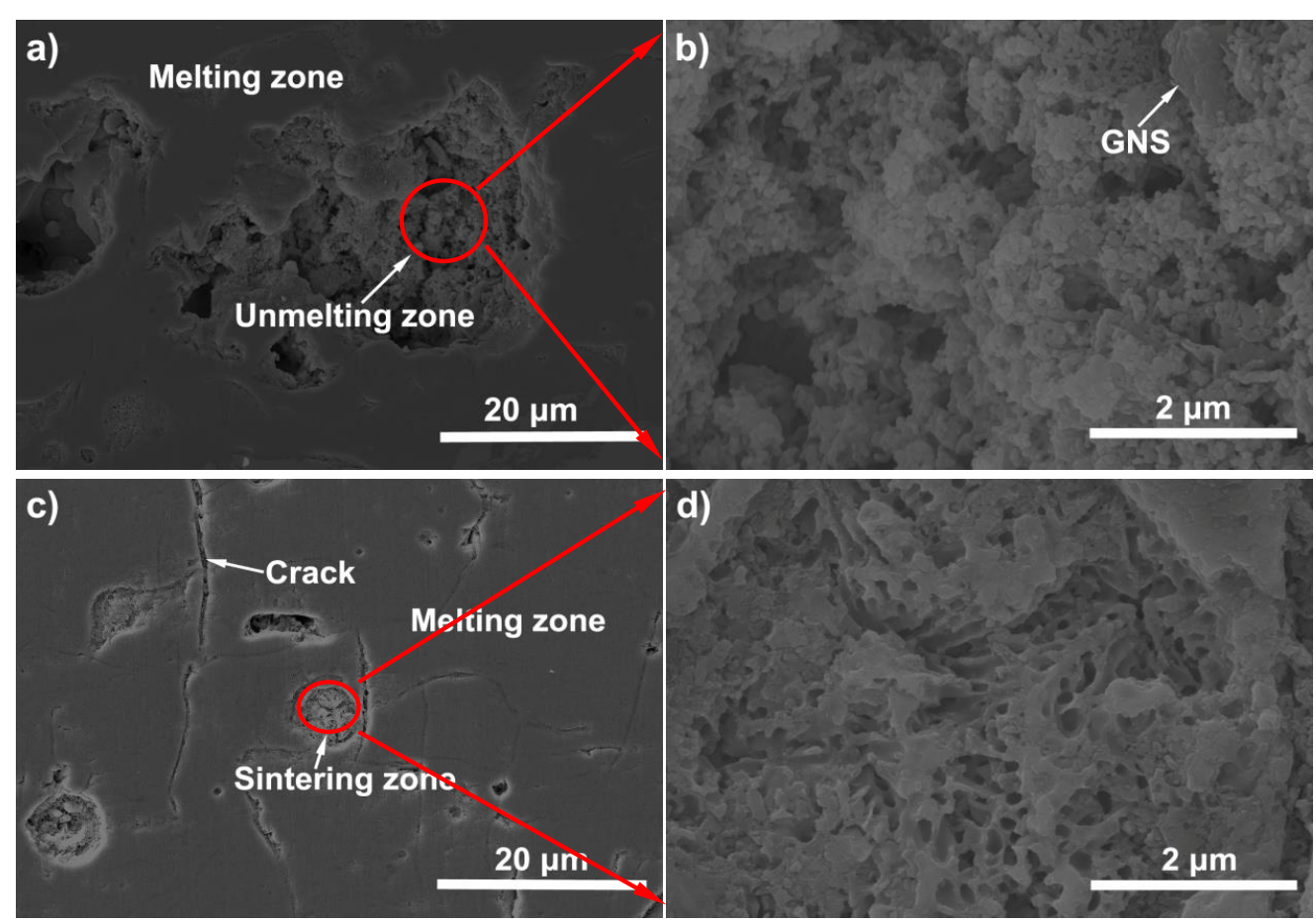

Figure 5. SEM cross-sectional microstructure of as-sprayed 1.0wt\%GNS/HA coating 
(a, b) and thermally treated $1.0 \mathrm{wt} \%$ GNS/HA coating (c, d).

To develop comprehensive understanding the contributions of the added GNSs to microstructure evolution of the thermally treated HA coatings, careful SEM observation was performed on these coatings with and without thermal treatment. It is evident from Figure 5a that the cross-section of the as-sprayed GNA/HA coating consisted of melted zones and partially melted/unmelted zones with porous characteristic, in which lots of nanoparticles and some GNSs were observed (Figure $5 b$, and the size of these nanoparticles was approximately equivalent to the as-received HA particles. The presence of partially melted/unmelted zones indicates that some HA particles inside the spray-dried agglomerated powders were not melted and remained in the as-sprayed coating, it should be related to the fact that the heat transfer toward the center of the agglomerates during plasma spray might be significantly limited due to these porous nanostructructed agglomerates used in this research. In contrast, the residual partially melted/unmelted zones of the thermally treated GNS/HA coating was significantly decreased in size and some microcracks were clearly seen, as depicted in Figure 5c. More importantly, the porous microstructure of the unmelted zone within the as-sprayed coating developed to more compact skeleton-like microstructure (Figure 5d), implying that effective sintering occurred in the process of thermal treatment. 

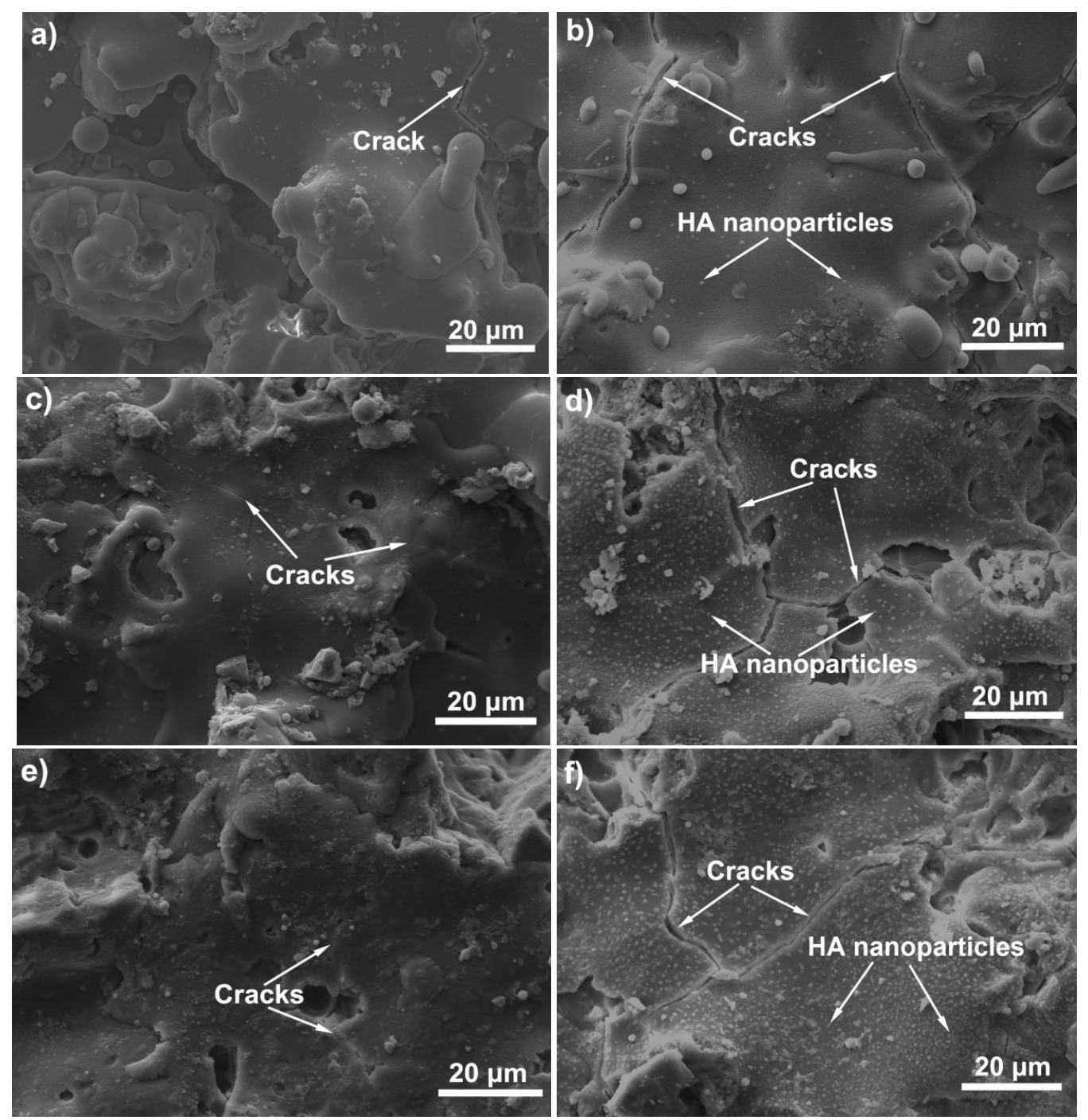

Figure 6. SEM images of surface morphology of (a) as-sprayed HA coating, (b) thermally treated HA coating, (c) as-sprayed 1.0wt\%GNS/HA coating, (d) thermally treated $1.0 \mathrm{wt} \%$ GNS/HA coating, (e) as-sprayed $2.0 \mathrm{wt} \% \mathrm{GNS} / \mathrm{HA}$ coating and (f) thermally treated $2.0 \mathrm{wt} \% \mathrm{GNS} / \mathrm{HA}$ coating.

Figure 6 shows the typical surface images of as-sprayed coatings with and without thermal treatment. It is evident from Figure 6a, $\mathrm{c}$ and e that the surfaces of these as-sprayed coatings consisted of partially melted and un-melted particles that were all adhered to the well melted splats, in which the small spherulites and dune-shaped particles corresponded to the un-melted and partially melted particles 
whereas smooth surfaces corresponded to fully melted splats. After thermal treatment, significant increase in the length of the microcracks was noticed (Figure $6 \mathrm{~b}, 6 \mathrm{~d}$ and 6f), which were in consistent with cross-sectional observations of the GNS/HA coating followed by thermal treatment (Figure 2c). Similar results have been reposted in previous research [38]. Once crystallisation and phase transformations occur in the process of thermal treatment, the stresses caused by the associated volume change would be superimposed on these microcracks already present [24], subsequently leading to the microcrack propagation. Furthermore, it is well known that crystalline structure is much more compact than that of the amorphous one, volume shrinkage along with ACP transformation into crystalline HA during thermal treatment would produce significant surface tensile stress, which in turn accelerates microcrack propagation in the process of thermal treatment. Most importantly, it is interesting to find that thermal treatment resulted in HA nanoparticle precipitation (Fig. 6b, d, f), especially HA nanoparticles almost pervaded throughout the top surfaces of the thermally treated GNS/HA coatings, which mainly ascribs to the transformation of by-products of plasma sprayed HA coatings (i.e., TCP, $\mathrm{CaO}$ ) to HA phase through hydrolytic reactions.

Figure 7 shows AFM images (capture area $10 \mu \mathrm{m} \times 10 \mu \mathrm{m}$ ) of the as-sprayed coatings before and after thermal treatment. The roughness factor of the as-sprayed HA coating following polishing was determined to be about root mean square (RMS) $=50.8 \pm 5.3 \mathrm{~nm}$, while $\mathrm{RMS}=65.0 \pm 7.5 \mathrm{~nm}$ for the as-sprayed $1.0 \mathrm{wt} \% \mathrm{GNS} / \mathrm{HA}$ coating and RMS $=69.5 \pm 8.4 \mathrm{~nm}$ for the as-sprayed $2.0 \mathrm{wt} \% \mathrm{GNS} / \mathrm{HA}$ coating. It is 
clear that the addition of GNSs slightly increased surface roughness of the as-sprayed coatings. Due to two-dimensional structure and the presence of obvious wrinkled texture of GNSs, these incorporated GNSs would lead to an increase in nanoscale topography of the as-sprayed HA coating. After thermal treatment, RMSs were measured to be $74.3 \pm 12.4 \mathrm{~nm}, 112.0 \pm 35.7 \mathrm{~nm}$ and $218.2 \pm 46.8 \mathrm{~nm}$ for HA coating, 1.0wt $\%$ GNS/HA coating and $2.0 \mathrm{wt} \%$ GNS/HA coating, respectively. Of particular importance was that the presence of added GNSs contributed greatly to the significant increase in surface roughness of the thermally treated HA coatings, for example, RMS of the thermally treated $2.0 \mathrm{wt} \% \mathrm{GNS} / \mathrm{HA}$ coating increased by up to $210 \%$ in comparison with that of the as-sprayed $2.0 \mathrm{wt} \% \mathrm{GNS} / \mathrm{HA}$ coating, which was also evidenced from the observation that HA nanoparticles precipitated throughout the surface of the GNS/HA coatings after thermal treatment (Figure 6d). These results strongly implied that the addition of GNSs is capable of accelerating retransformation of crystalline HA during thermal treatment. As revealed in Raman spectra (Figure 5b), thermal treatment induced much more structural defects into the added GNSs within the as-sprayed HA composite coatings, and therefore it is hypothesized that these structural defects of the GNSs might act as nucleation sites to accelerate HA nanoparticle precipitation in the process of thermal treatment, giving rise to the significant increase in the surface roughness on the surface of the thermally treated composite coatings. The presence of the nanozones on the thermally treated GNS/HA composite coatings would act as anchors to interact with nanosized adhesion proteins such as fibronectin $(\sim 150 \mathrm{~nm})$ [39], which would be beneficial for the improved 
adhesion strength of the osteoblast cells on the coating surface.

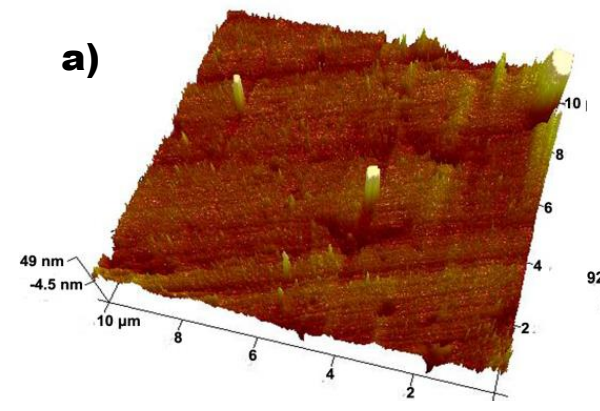

RMS $=50.8 \pm 5.3 \mathrm{~nm}$

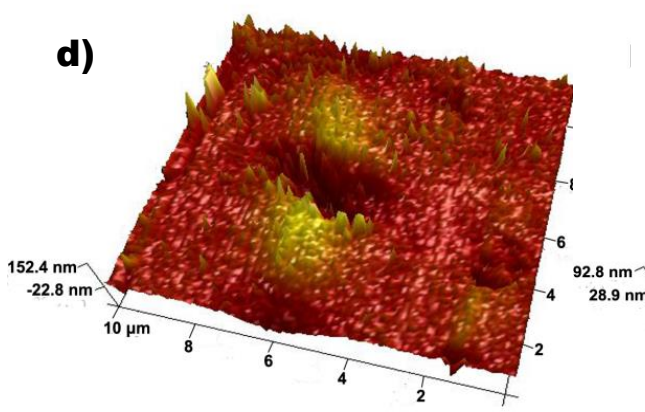

$\mathrm{RMS}=74.3 \pm 12.4 \mathrm{~nm}$

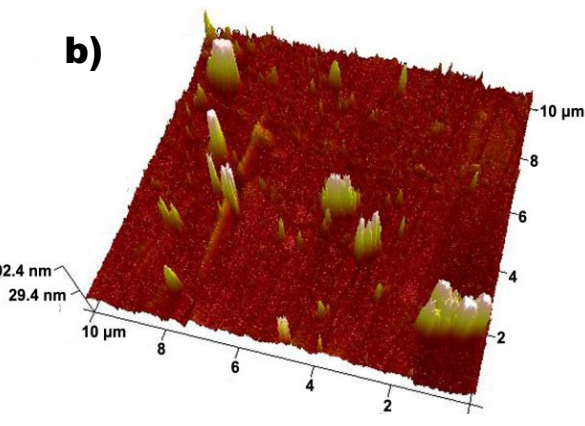

$\mathbf{R M S}=65.0 \pm 7.5 \mathrm{~nm}$

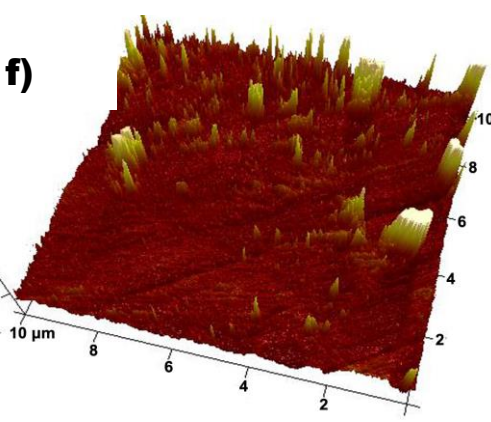

RMS $=112.0 \pm 35.7 \mathrm{~nm}$

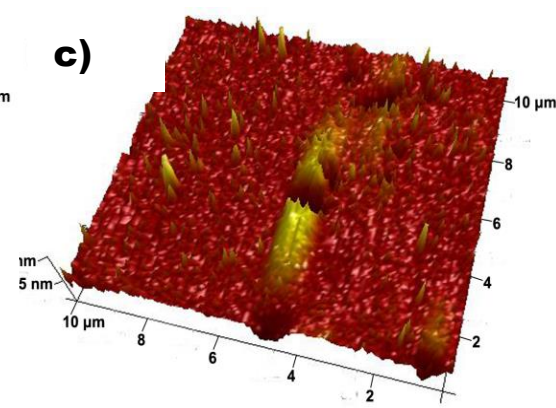

RMS=69.5 $\pm 8.4 \mathrm{~nm}$

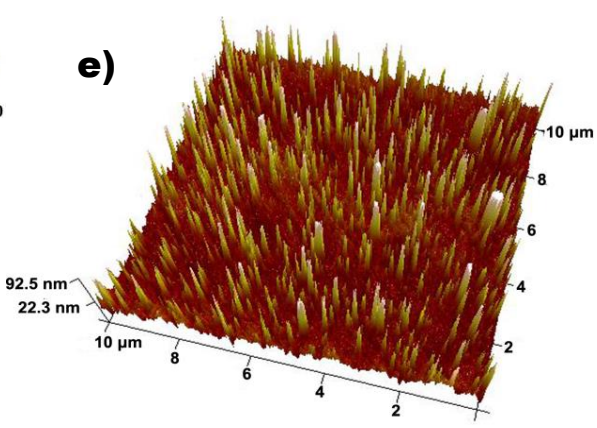

RMS $=218.2 \pm 46.8 \mathrm{~nm}$

Figure 7. AFM images of (a) as-sprayed HA coating, (b) as-sprayed 1.0wt\%GNS/HA coating, (c) as-sprayed 2.0wt\%GNS/HA coating, (d) thermally treated HA coating, (e) thermally treated $1.0 \mathrm{wt} \% \mathrm{GNS} / \mathrm{HA}$ coating and (f) thermally treated $2.0 \mathrm{wt} \% \mathrm{GNS} / \mathrm{HA}$ coating.

\section{Conclusions}

Monolithic HA and graphene nanosheets (GNS) reinforced HA coatings were fabricated on the Ti6Al4V using plasma spray, and then they were subjected to heat and hydrothermal treatment. During plasma spray, HA partially decomposed and also formed amorphous calcium phosphate (ACP), leading to low degree of HA crystallinity ( 46.0-51.3\%). Also, HA crystallinity in the as-sprayed coating slightly increased with the added GNSs due to the fact that these GNSs resulted in competing phenomenon to influence HA crystallinity viz. increased portion of the partially 
melted/unmelted zones and higher cooling rate during splat formation. After heat and hydrothermal treatment, HA coatings displayed $~ 45.5-47.1 \%$ improvements in HA crystallinity regardless of addition or not of the GNS nanofillers. Above results illustrated that heat and hydrothermal treatment was capable of inducing significant transformation of amorphous HA to the crystalline form and removing other foreign non-HA compounds through regaining $\mathrm{OH}^{-}$ion. Moreover, heat and hydrothermal treatment not only made the as-sprayed HA coating more compact in virtue of effective sintering of partially melted/unmelted zones present in the as-sprayed coatings, but also facilitated microcrack propagation due to stresses caused by crystallisation and phase transformations. Raman spectra revealed that heat and hydrothermal treatment induced much more structure defects of the added GNSs, which might act as nucleation sites to accelerate HA nanoparticle precipitation during heat and hydrothermal treatment, subsequently leading to the significant increase in surface roughness of the thermally treated HA coatings ( $210 \%$ increase in RMS of the $2.0 \mathrm{wt} \% \mathrm{GNS} / \mathrm{HA}$ coating followed by heat and hydrothermal treatment). The presence of nanozones of the GNS/HA coatings would be beneficial for the improved adhesion strength of the osteoblast cells on their surface.

Author Contributions: Conceptualization, Y.C.; Methodology, J.R., D.Z., and F.Q.; Validation, J.R., D.Z., Y.G.W., and Y.C.; Analysis, J.R., D.Z., F.Q.; Investigation, J.R., Y.G.W.; Resources, J.R., Y.C.; Data Curation, J.R.; Writing-Original Draft Preparation, J.R.; Writing-Review \& Editing, Y.G.W., Y.C.; Supervision, Y.C.; Project Administration, Y.C.; Funding Acquisition, Y.C. 
Funding: The research was funded by the National Natural Science Foundation of China $(51471113,51275326)$ and the Natural Science Foundation of Jiangsu Province, China (BK20140301).

Acknowledgments: The authors thank the anonymous reviewers for their constructive comments.

Conflicts of Interest: The authors declare no conflicts of interest.

\section{References}

[1] Hench, L.L. Bioceramics: From concept to clinic. J. Am. Ceram. Soc. $1991,74,1487-1510$.

[2] Jarcho, M. Calcium phosphate ceramics as hard tissue prosthetics, Clin. Orthop. Relat. Res. 1981,157,259-278.

[3] Joshi, S.V.; Srivastava, M.P.; Pal, A.; Pal, S. Plasma spraying of biologically derived hydroxyapatite on implantable materials. J. Mater. Sci. Mater. Med. 1993,4, $251-255$.

[4] Kumar, A.; Biswas, K.; Basu, B. On the toughness enhancement in hydroxyapatite-based composites. Acta Mater. 61(2013) 5198-5215.

[5] Wennerberg, A.; Albrektsson,T. Structural influence from calcium phosphate coatings and its possible effect on enhanced bone integration. Acta Odontol. Scand. 2009,67,333-340.

[6] Manara, S.; Paolucci, F.; Palazzo, B.; Marcaccio, M.; Foresti, E.; Tosi, G.; Sabbatini, S.; Sabatino, P.; Altankov, G.; Roveri, N. Electrochemically-assisted 
deposition of biomimetic hydroxyapatite-collagen coatings on titanium plate. Inorg. Chim. Acta 2008,361,1634-1645.

[7] Bajpai, S.; Gupta, A.; Pradhan, S.K.; Mandal, T.; Balani, K. Crack Propagation Resistance of alpha- $\mathrm{Al}_{2} \mathrm{O}_{3}$ reinforced pulsed laser-deposited hydroxyapatite coating on 316 stainless steel. JOM 2014,66,2095-2107.

[8] Mittal, M.; Nath, S.K.; Prakash, S. Improvement in mechanical properties of plasma sprayed hydroxyapatite coatings by $\mathrm{Al}_{2} \mathrm{O}_{3}$ reinforcement. Mater. Sci. Eng. C $2013,33,2838-2845$.

[9] Fu, L.; Khor, K.A.; Lim, J.P. Processing, microstructure and mechanical properties of yttria stabilized zirconia reinforced hydroxyapatite coatings. Mater. Sci. Eng. A 2001,316,46-51.

[10] Fu, L.; Khor, K.A.; Lim, J.P. Effects of yttria-stabilized zirconia on plasma-sprayed hydroxyapatite/yttria-stabilized zirconia composite coating. J. Am. Ceram. Soc. 2002,85,800-806.

[11] Yugeswaran, S.; Kobayashi, A.; Ucisik, A.H.; Subramanian, B. Characterization of gas tunnel type plasma sprayed hydroxyapatite-nanostructure titania composite coatings. Appl. Surf. Sci. 2015,347,48-56.

[12] Lu, Y.P.; Li, M.S.; Li, S.T.; Wang, Z.G.; Zhu, R.F. Plasma-sprayed hydroxyapatite +titania composite bond coat for hydroxyapatite coating on titanium substrate. Biomater. 2004,25,4393-4403. 
[13] Zhu, J.Y.; Chen, Y.; Ren, J.; Zhao, D.; Liu, W.W. Boron nitride nanoplatelets induced synergetic strengthening and toughening effects on splats and their boundaries of plasma sprayed hydroxyapatite coatings. Ceram. Int. 2018,44,10604-10. [14] Zhan, G.D.; Kuntz, J.D.; Wan, J.; Mukherjee, A.K. Single-walled carbon nanotubes as attractive toughening agents in alumina based nanocomposites. Nat. Mater. 2003,2,38-42.

[15] M.A. Rafiee, J. Rafiee, . Wang, H.H. Song, Z.Z. Yu, N. Koratkar, Enhanced mechanical properties of nanocomposites at low graphene content, ACS Nano 3(2009)3884-3890.

[16] Balani, K.; Anderson, R.; Laha, T.; Andara, M.; Tercero, J.; Crumpler, E.; Agarwal, A. Plasma-sprayed carbon nanotube reinforced hydroxyapatite coatings and their interaction with human osteoblasts in vitro. Biomater. 2007,28,618-624.

[17] Balani, K.; Chen, Y.; Harimkar, S.P.; Dahotre, N.B.; Agarwal. A. Tribological Behavior of Plasma Sprayed HA Composite Coating Reinforced with Carbon Nanotubes, Acta Biomater. 2007,3,944-951.

[18] Lahiri, D.; Ghosh, S.; Agarwal, A. Carbon nanotube reinforced hydroxyapatite composite for orthopedic application: a review. Mater. Sci. Eng. C $2012,32,1727-1758$.

[19] Park, S.; Ruoff, R.S. Chemical methods for the production of graphenes. Nat. Nanotechnol. 2009,4,217-24.

[20] Zhang, L.; Liu, W.W.; Yue, C.G.; Zhang, T.H.; Li, P.; Xing, Z.W.; Chen. Y. A tough graphene nanosheet/hydroxyapatite composite with improved in vitro 
biocompatibility. Carbon 2013, 61,105-115.

[21] Zhang, L.; Zhang, X.G.; Chen, Y.; Su, J.N.; Liu, W.W.; Zhang, T.H.; Qi, F.; Wang. Y.G. Interfacial stress transfer in a graphene nanosheet toughened hydroxyapatite composite. Appl. Phys. Lett. 2014,105,161908.

[22] Gross, K.A.; Berndt, C.C. Thermal processing of hydroxyapatite for coating production. J. Biomed. Mater. Res. 1998,39,580-587.

[23] Hartmann, P.; Jager, C.; Barth, S.; Vogel, J.; Meyer, K. Solid state NMR, X-ray diffraction, and infrared characterization of local structure in heat-treated oxyhydroxyapatite microcrystals: an analog of the thermal decomposition of hydroxyapatite during plasma-spray procedure. J. Solid Sate Chem. $2001,160,460-468$.

[24] Tsui, Y.C.; Doyle, C.; Clyne, T.W. Plasma sprayed hydroxyapatite coatings on titanium substrates Part 2: optimisation of coating properties. Biomater. 1998,19, 2031-2043.

[25] Y.P. Lee, C.K. Wang, T.H. Huang, C.C. Chen, C.T. Kao, S.J. Ding, In vitro characterization post heat-treated plasma-sprayed hydroxyapatite coatings, Surf. Coat. Technol. 197(2005) 367.

[26] Xiao, G.Y.; Lu, Y.P.; Zhu, R.F.; Li, S.T.; Wang, A.J. Effect of post-deposition heat treatment on mechanical properties of thermally sprayed hydroxyapatite coating. Surf. Eng. 2008,24,307-312.

[27] Lu, Y.P.; Song, Y.Z.; Zhu, R.F.; Li, M.S. Factors influencing phase compositions and structure of plasma sprayed hydroxyxapatite coatings during heat treatment. Appl. 
Surf. Sci. 2003,206,345-354.

[28] Lu, Y.P.; Chen, Y.M.; Li, S.T.; Wang, J.H. Surface nanocystallization of hydroxyxapatite coating. Acta Biomater. 2008,4,1865-1872.

[29] Tian , Y.S.; Qian, X.L.; Chen, M.Q. Effect of saturated steam treatment on the crystallinity of plasma-sprayed hydroxyapatite coatings. Surf. Coat. Technol. $2015,266,38-41$

[30] Zhu, J.Y.; Ren, J.; Chen, Y. Splat-boundary reinforcement induced by graphene in plasma sprayed hydroxyapatite composite coating. Carbon Lett. In press.

[31]A. Singh, G. Singh, V. Chawla, Characterization of vacuum plasma sprayed reinforced hydroxyapatite coatings on Ti-6Al-4V alloy, Trans. Indian Institute Metals, 70(2017)2609-2628.

[32] Balandin, A.A.; Ghosh, S.; Bao, W.; Calizo, I.; Teweldebrhan, D.; Miao, F.; Lau, C.N. Superior thermal conductivity of single-layer graphene. Nano letters 2008,8, 902-907.

[33] Ferrari, A.C.; Meyer, V.; Scardaci, J.C.; Casiraghi, C.; Lazzer, M.; Mauri, F.; Piscanec, S.; Jiang, D.; Novoselov, K.S.; Roth, S.; Geim, A.K. Raman spectrum of graphene and graphene layers. Phys Rev Lett, 2006,97,187401.

[34] Ni, Z.H.; Wang, H.M.; Ma, Y.; Kasim, J.; Wu, Y.H.; Shen, Z.X. Tunable stress and controlled thickness modification in graphene by annealing. ACS Nano 2008,2,1033-1039.

[35] Inam, F, Vo, T; Bhat, B.R. Structural stability studies of graphene in sintered ceramic nanocomposites. Ceram. Inter. 2014,40,16227-16233. 
[36] Fan, Z.J.; Wang, J.Q.; Wang, Z.F.; Ran, H.Q.; Li, Y.; Niu, P L.; Gong, Y.W.; Liu, B.; Yang, S.R. One-pot synthesis of graphene/hydroxyapatite nanorod composite for tissue engineering. Carbon 2014,66,407-416.

[37] Chen, J.Y.; Tong, W.D.; Cao, J.; Feng, Y.M.; Zhang, X.D. Effect of atmosphere on phase transformation in plasma-sprayed hydroxyapatite coatings during heat treatment. J. Biomed. Mater. Res. 1997,34,15-20.

[38] Singh, Singh, A.G.; Chawla, V. Influence of post coating heat treatment on microstructural, mechanical and electrochemical corrosion behaviour of vacuum plasma sprayed reinforced hydroxyapatite coatings. J. Mech. Behav. Biomed. Mater. 2018,85,20-36.

[39] Erickson, H.P.; Carrell, N.; McDonagh, J. Fibronectin molecure visuallized in electron microcopy: A long, thin, felxible strand. J. Cell Biol. 1981,91,673-678. 